\title{
Un análisis descriptivo de la arquitectura sintáctica en la oralidad ${ }^{\star}$
}

\author{
Ricardo Benítez $z^{* *}$ Carola Alvarado
}

\begin{abstract}
Resumen
Este artículo presenta los resultados de un estudio de casos que sirvió como prueba de pilotaje para una investigación mayor. Para tal efecto, se exploró la complejidad sintáctica en niños que cursan diferentes niveles de escolaridad, determinándose el porcentaje promedio de aparición de las relaciones sintácticas en la producción oral de cada uno de los grupos etarios. El análisis consideró las estructuras sintácticas producidas en el desarrollo de dos secuencias textuales: narración y explicación, con la finalidad de estudiar qué estructuras emergen en mayor o menor grado en cada uno de los niveles de escolaridad estudiados. La muestra estuvo conformada por 38 estudiantes chilenos que cursan kinder, segundo básico, cuarto básico, octavo básico y cuarto medio. El trabajo ha permitido determinar tanto qué estructuras ocurren en los primeros años escolares y cuáles emergen en los años posteriores así como su frecuencia de uso.
\end{abstract}

Palabras clave: complejidad sintáctica; producción oral; narración y explicación.

\section{A descriptive analysis of the syntactic architecture of speaking}

\begin{abstract}
This article shows the results of a pilot case study which was later used in an extended research. The study explored the syntactic complexity produced by school children from different levels in order to determine the average percentage of syntactic relations in their speaking, per age. The analysis took into account the syntactic structures produced in the development of two types of text sequences: narrative and explanation to find out which structures are more frequently or less frequently produced in the different school levels studied. The sample consisted of 38 Chilean students from foundation, second, fourth and eighth year of primary school, and from fourth year of secondary school. This work helped determine which structures occur during the first years of schooling and which ones are produced in further years as well as their use frequencies.
\end{abstract}

Key words: Syntactic Complexity; Speaking; Narrative and Explanation

\footnotetext{
Proyecto del Fondo para la Educación, Ciencia y Tecnología de Chile (FONDECYT) $\mathrm{N}^{\circ} \mathrm{II} 100600$.

* Pontificia Universidad Católica de Valparaíso.rbenitez@ucv.cl

Pontificia Universidad Católica de Valparaíso.carola_alvarado@mi.cl
} 


\section{Introducción}

Un evidente cambio de perspectiva en los estudios de la complejidad sintáctica se origina desde que estos se enfocaban en el análisis de la producción escrita hasta la actual inquietud de analizar la producción oral. Estos últimos trabajos (Berman, 1996, 2004; Katzenberger, 1999, 2003; Nir-Sagiv y Berman, 2010), se caracterizan por ser translingüísticos en al menos cinco idiomas y no se centran en el español propiamente tal, a excepción del trabajo sobre la alternancia de los agentes como recurso retórico en las construcciones pasivas (Tolchinsky \& Rosado, 2005). Tradicionalmente, las investigaciones sobre la sintaxis de la oralidad han logrado grandes avances, especialmente respecto a la adquisición inicial de este ámbito del lenguaje. No obstante, lo que sucede con su desarrollo tardío ha sido descrito con menos profundidad, sin especificarse cómo va ocurriendo ni cómo va afectando la escolaridad. Estudios recientes (Katzenberger, 2003; Levy; 2003; Berman, 2004; Coloma, Peñaloza y Fernández, 2007; Nir-Sagiv y Berman, 2010) se han caracterizado por investigar la sintaxis oral desde un ámbito más formal en distintas modalidades discursivas.

En Chile, los trabajos referidos a la complejidad sintáctica (Véliz, I988, 1999) se han basado principalmente en los análisis realizados por Hunt (1970). Este autor propuso un índice para medir la madurez sintáctica a partir de una unidad denominada Unidad-T. Ésta se concibió como la unidad mínima terminal que permite calcular el promedio de longitud de una cláusula principal, considerando todas las otras cláusulas incrustadas en ella o adjuntadas a ella. Si bien dichos estudios han contribuido a desarrollar investigaciones de la complejidad sintáctica, no han adoptado un enfoque funcionaldiscursivo como el que adoptan las más recientes (Berman, I996; Katzenberger, 1999), las cuales han permitido transformar la visión de lo que se denomina 'madurez sintáctica'. Ésta se consideraba como una cadena de cláusulas principales y subordinadas; sin embargo, en la actualidad, este concepto se entiende como una construcción de relaciones de significados al interior de unidades mayores. Es decir, el estudio de la madurez sintáctica se centra ahora en lo coherente de las estructuras sintácticas en su relación con el tema tratado o con su función lingüística. 
De esta manera, en el presente estudio no se utiliza ni el concepto de oración ni el de párrafo. De acuerdo con Nir-Sagiv y Berman (2010), la oración es un término gramatical abstracto, un ideal de la gramática normativa, el cual se ve limitado por un modelo formal particular de sintaxis; asimismo, la oración se considera como una unidad en contextos lingüísticos susceptibles de ser editados. Por otra parte, párrafo se entiende como unidad de discurso que no ha sido analizado en forma empírica o no obedece a definiciones psicolingüísticamente motivadas (Heurly, 1997). Además, la oración y el párrafo se asocian conceptualmente con la escritura más que con la oralidad. Lo anterior proporciona fundamentos para que en el presente trabajo no se consideren estos conceptos, pues se analizan textos orales producidos por hablantes-escritores inexpertos, los cuales no están ni corregidos con propósitos editoriales, ni analizados bajo una matriz sintáctica determinada.

El presente trabajo constituye un análisis de las estructuras sintácticas en dos modalidades discursivas -la narración y la explicaciónproducidas oralmente por estudiantes chilenos de diferentes niveles escolares. Lo anterior, con la finalidad de estudiar qué estructuras emergen en mayor o menor grado en cada uno de los niveles de escolaridad estudiados. De esta forma, se asume que los niños no utilizan las mismas estructuras sintácticas para la producción de discursos orales narrativos que para la producción de discursos orales explicativos. Además, con este estudio se podrá determinar tanto qué estructuras ocurren en los primeros años escolares y qué estructuras emergen en los años posteriores así como su frecuencia de uso.

\section{Marco Teórico}

\subsection{Secuencia textual y paquete clausular}

Cahana-Amitay y Sandbank (2000) postulan una forma de descripción de las relaciones sintácticas a partir de lo que denominan 'paquetes clausulares' (PC). Éstos están conformados por cláusulas que se definen como unidades que presentan un predicado unificado que expresa una situación única (actividad, evento o estado (Berman y Slobin, 1994). Los PC se describen como las unidades textuales 
básicas compuestas por varias cláusulas vinculadas, ya sea por estándares sintácticos, temáticos y/o discursivos (Nir-Sagiv y Berman, 2010). Bajo estos criterios, un PC es una unidad textual que trasciende una arquitectura sintáctica particular, como por ejemplo la cláusula, la oración, o la Unidad-T. Además, trasciende ciertos elementos discursivos, como las 'unidades idea' de Chafe (1994), las 'unidades temáticas' de Scinto (1984) o las 'estrofas' de Gee (1989). De esta forma, dentro de la mayoría de los PC las relaciones se presentan explícitamente marcadas por conjunciones paratácticas o hipotácticas, aunque también, en ciertas ocasiones, la progresión temática del texto permite inferirlas. Por otro lado, en el disucrso oral un PC se marca a partir de la entonación, los silencios, las pausas, el tono, el ritmo y el acento, es decir, los rasgos prosódicos del discurso.

Este estudio intenta describir la complejidad sintáctica en dos tipos de secuencias textuales (narrativa y explicativa) a través del análisis de PC. Por lo general, la madurez sintáctica ha sido determinada por la cantidad de relaciones tanto subordinadas como coordinadas que se presentan dentro de un texto escrito. Bartolomé (2008) sostiene que, bajo esta perspectiva, una persona es más madura sintácticamente mientras mayor sea el número de relaciones sintácticas que emplea para producir discurso.

Con respecto a la secuencia textual, Adam (1992, 1996) plantea que ésta es una red de relaciones jerárquicas, una totalidad que se puede descomponer en partes relacionadas entre sí y con el todo. Una entidad relativamente autónoma dotada de una organización interna que le es propia. La secuencia se ubica dentro de la estructura del texto, constituye una unidad textual que tiene un conjunto de propiedades y está formada por proposiciones. En este sentido, un texto estaría consitutido por secuencias, las secuencias por macroproposiciones y éstas por microproposiciones. Dependiendo del tipo de secuencia que predomina, se puede establecer a qué tipología textual pertenece dicho texto. Así, este autor propone cinco tipos de secuencias textuales: narrativa, descriptiva, argumentativa, explicativa, y dialogal. En este trabajo, sin embargo, se estudian sólo las secuencias narrativa y explicativa. 


\subsection{Clasificación de estructuras sintácticas}

A continuación, se presenta una propuesta de clasificación de construcciones sintácticas que ocurren en PC. Dicha clasificación se basa en los resultados de la investigación realizada por Nir-Sagiv y Berman (2010), específicamente en los aportes que arrojó su análisis de los tipos de relaciones intraclausulares detectadas en la producción oral de su muestra, la cual estaba compuesta por niños, adolescentes y universitarios que hablaban cinco idiomas diferentes y que cursaban diversos niveles de escolaridad. Esta clasificación ha surgido a partir del análisis de 38 participantes que componen la muestra de la presente investigación. El siguiente orden de las relaciones sintácticas sugiere más o menos el mismo orden en que aparecen al analizar un $\mathrm{PC}^{\mathrm{I}}$ :

- Cláusulas isotácticas. Son aquellas que aparecen en forma independiente y son asindéticas: no se vinculan a las cláusulas que la acompañan y no se encuentran conectadas por medio de una conjunción. Por lo general, pueden presentarse en dos formas: (a) cláusula isotáctica única [ISOuni], con total aislamiento del resto de las cláusulas; y (b) cláusulas isotácticas principales [ISOprin], introducciones de cada PC e isotácticas en relación a las otras ISOprin de los PC adyacentes.

\begin{tabular}{|l|l|l|}
\hline TIPO & & EJEMPLOI ${ }^{2}$ \\
\hline Isotaxis única & {$[$ ISOuni $]$} & Un extraterrestre cayó en la... el planeta Tierra. \\
\hline $\begin{array}{l}\text { Isotaxis } \\
\text { principal }\end{array}$ & {$[$ ISOprin $]$} & $\begin{array}{l}\text { Las escuelas existen porque antes en las épocas anteriores } \\
\text { eh los papás le enseñaban todo a sus hijos. }\end{array}$ \\
\hline
\end{tabular}

- Cláusulas paratácticas simétricas: Son aquellas que se relacionan a un mismo nivel sintáctico y se presentan de manera encadenada. Pueden encontrarse de dos formas: yuxtaposición y coordinación. La primera no es marcada sintácticamente (asidéntica), pero se relaciona con las otras cláusulas semántica y pragmáticamente; la segunda explicita su marcación a través de conjunciones y pueden

I En [ ] se indican las abreviaturas empleadas en el análisis.

2 Los ejemplos fueron extraídos de las producciones orales analizadas y no se han modificado para su reproducción en este trabajo. Los segmentos en cursiva son los que corresponden a la clasificación aludida. 
ocurrir con un sujeto implícito o explícito. El presente análisis arrojó la siguiente subclasificación de la parataxis simétrica:

\begin{tabular}{|c|c|c|}
\hline TIPO & & EJEMPLO \\
\hline $\begin{array}{l}\text { Yuxtapuestas c/ sujeto } \\
\text { idéntico explícito }\end{array}$ & [SIMyuxSIEx] & $\begin{array}{l}\text { Al principio, no... no quería hacer } \\
\text { daño, bueno él no quiere }\end{array}$ \\
\hline $\begin{array}{l}\text { Yuxtapuestas c/ sujeto } \\
\text { idéntico elíptico }\end{array}$ & [SIMyuxSIEl] & $\begin{array}{l}\text { Eh... un día Xentío Iofe se } \\
\text { empezó... se empezó a poner azul, } \\
\text { tenía que comer aloo. }\end{array}$ \\
\hline $\begin{array}{l}\text { Yuxtapuestas c/ sujeto } \\
\text { diferente explícito }\end{array}$ & [SIMyuxSDEx] & $\begin{array}{l}\text { Él intenta decir "hola", todo... } \\
\text { nadie responde. }\end{array}$ \\
\hline $\begin{array}{l}\text { Yuxtapuestas c/ sujeto } \\
\text { diferente elíptico }\end{array}$ & [SIMyuxSDEl]. & $\begin{array}{l}\text { Y llega un marciano extrañamente } \\
\text { y les cambia todo, le enseñan }\end{array}$ \\
\hline $\begin{array}{l}\text { Coordinadas c/ sujeto } \\
\text { idéntico explícito }\end{array}$ & [SIMcoordSIEx] & $\begin{array}{l}\text { Y después vieron una luz y todos } \\
\text { estaban asustados. }\end{array}$ \\
\hline $\begin{array}{l}\text { Coordinadas c/ sujeto } \\
\text { idéntico elíptico }\end{array}$ & [SIMcoordSIEl] & $\begin{array}{l}\text { Y un día se puso azul y le costaba } \\
\text { bablar }\end{array}$ \\
\hline $\begin{array}{l}\text { Coordinadas c/ sujeto } \\
\text { diferente explícito }\end{array}$ & {$[$ SIMcoordSDEx } & $\begin{array}{l}\text { Hasta que le salió el "hola" y los } \\
\text { presentes quedaron asombrados }\end{array}$ \\
\hline $\begin{array}{l}\text { Coordinadas c/ sujeto } \\
\text { diferente elíptico }\end{array}$ & [SIMcoordSDEl] & $\begin{array}{l}\text { Nadie entendía qué decía y después } \\
\text { dijo "bo }\end{array}$ \\
\hline
\end{tabular}

- Cláusulas paratácticas asimétricas. Son aquellas que presentan una relación dependiente de la cláusula que la precede. Por esta razón, este tipo de estructura adquiere significado sólo a partir de la anterior. En el análisis se describen tres tipos de relaciones paratácticas asimétricas:

\begin{tabular}{|l|l|l|}
\hline TIPO & & EJEMPLO \\
\hline $\begin{array}{l}\text { Yuxtapuesta c/ verbo } \\
\text { idéntico elíptico }\end{array}$ & [ASIMyuxvie $]$ & $\begin{array}{l}\text { Justo en un día se apareció una luz } \\
\text { incandescente, una luz celeste. }\end{array}$ \\
\hline $\begin{array}{l}\text { Coordinada con verbo } \\
\text { idéntico elíptico }\end{array}$ & {$[$ ASIMcoordvie $]$} & $\begin{array}{l}\text { Hasta que apareció una especie de } \\
\text { nave donde una rara criatura que } \\
\text { era verde que no era ni bumano ni un } \\
\text { ser ni un animal. }\end{array}$ \\
\hline $\begin{array}{l}\text { Subordinada sustantiva } \\
\text { de objeto directo } / \\
\text { complemento }\end{array}$ & {$[$ ASIMcomp $]$} & $\begin{array}{l}\text { Todos comenzaron a aplaudir } \\
\text { emocionados y felices, ya que } \\
\text { sabían que se podían comunicar con ese } \\
\text { extraterrestre. }\end{array}$ \\
\hline
\end{tabular}

- Cláusulas hipotácticas: Son aquellas que se relacionan con la cláusula anterior de manera dependiente de ésta, a través de una conjunción subordinante. En este análisis, se detectaron diez clases de hipotaxis: 


\begin{tabular}{|c|c|c|}
\hline TIPO & & EJEMPLO \\
\hline Hipotácticas adjetivas & [HIPOadj] & $\begin{array}{l}\text { Y que era un extraterrestre que } \\
\text { flotaba sobre el piso. }\end{array}$ \\
\hline $\begin{array}{l}\text { Hipotácticas adjetivas } \\
\text { c/ verboide }\end{array}$ & [HIPOadjverboi] & $\begin{array}{l}\text { Y porque antes no había como } \\
\text { tantas... como tantas cosas que } \\
\text { aprender. }\end{array}$ \\
\hline $\begin{array}{l}\text { Hipotácticas } \\
\text { sustantivas }\end{array}$ & [HIPOsus] & $\begin{array}{l}\text { Y bueno lo que más le gustaba era } \\
\text { el recreo. }\end{array}$ \\
\hline $\begin{array}{l}\text { Hipotácticas } \\
\text { sustantivas c/ verboide }\end{array}$ & [HIPOsusverboi] & $\begin{array}{l}\text { Y entonces se dio cuenta que } \\
\text { era hora de regresar. }\end{array}$ \\
\hline $\begin{array}{l}\text { Hipotácticas } \\
\text { adverbiales propias }\end{array}$ & {$[\mathrm{HIPO}$ advPR $]$} & $\begin{array}{l}\text { Él aún no conocía bien } \\
\text { nuestro idioma basta que él se } \\
\text { comienza a acordar. }\end{array}$ \\
\hline $\begin{array}{l}\text { Hipotácticas } \\
\text { adverbiales propias c/ } \\
\text { verboide }\end{array}$ & [HIPOadvPRverboi] & Y sin decir adiós se fue. \\
\hline $\begin{array}{l}\text { Hipotácticas } \\
\text { adverbiales impropias } \\
\text { consecutivas }\end{array}$ & [HIPOadvIMPconsec] & $\begin{array}{l}\text { Avanza tan rápidamente que los } \\
\text { padres ya no pueden entregarle todo e } \\
\text { conocimiento nuevo a sus bijos. }\end{array}$ \\
\hline $\begin{array}{l}\text { Hipotácticas } \\
\text { adverbiales impropias } \\
\text { causales }\end{array}$ & [HIPOadvIMPcausal] & $\begin{array}{l}\text { Bueno las escuelas son } \\
\text { importantes porque abi aprenden. }\end{array}$ \\
\hline $\begin{array}{l}\text { Hipotácticas } \\
\text { adverbiales impropias } \\
\text { finales }\end{array}$ & [HIPOadvIMPfinal] & $\begin{array}{l}\text { Le enseñaban a leer, los } \\
\text { cuidaban para que no estuvieran } \\
\text { solos en su casa. }\end{array}$ \\
\hline $\begin{array}{l}\text { Hipotácticas } \\
\text { adverbiales impropias } \\
\text { condicionales }\end{array}$ & [HIPOadvIMPcondic] & $\begin{array}{l}\text { Porque si no bubieran escuelas los } \\
\text { niños no podrían aprender nada. }\end{array}$ \\
\hline
\end{tabular}

- Cláusulas endotácticas. Son aquellas que se ubican dentro de otra cláusula y se manifiestan como una anidación de cláusulas. En este análisis, se describen seis clases de endotaxis:

\begin{tabular}{|l|l|l|}
\hline TIPO & EJEMPLO \\
\hline Endotaxis adjetiva & {$[$ ENDOadj] } & $\begin{array}{l}\text { Y nos enseñan los profesores, que } \\
\text { son inteligentes. }\end{array}$ \\
\hline Endotaxis sustantiva & {$[$ ENDOsus $]$} & $\begin{array}{l}\text { Lo que él hacía que organizaba viajes } \\
\text { a la luna. }\end{array}$ \\
\hline Endotaxis adverbial & {$[$ ENDOadv $]$} & $\begin{array}{l}\text { La nave llegó allí, donde estaban los } \\
\text { niños. }\end{array}$ \\
\hline Endotaxis parentética & {$[$ ENDOparent $]$} & $\begin{array}{l}\text {...hasta que el marciano en la lengua de } \\
\text { Xentío Iofe... }\end{array}$ \\
\hline Endotaxis reparadora & {$[$ ENDOrep $]$} & \begin{tabular}{l} 
ehh...ehh... qué más a ver \\
\hline
\end{tabular} \\
\hline
\end{tabular}


- Nominalización [Nom]. Es un recurso sintáctico que se utiliza como metáfora gramatical (Halliday, I993; Thompson, 2004), la cual es un enunciado que deriva desde una estructura gramatical congruente (generalmente un verbo) hacia una forma gramatical nueva (generalmente un sustantivo).

\begin{tabular}{|l|l|l|}
\hline TIPO & & EJEMPLO \\
\hline Nominalización & {$[$ Nom $]$} & $\begin{array}{l}\text { Las escuelas existen por la necesidad } \\
\text { de aprender... }\end{array}$ \\
\hline
\end{tabular}

\section{Metodología}

La presente investigación es de carácter cuantitativo descriptivo, pues los objetivos son, por una parte, explorar la complejidad sintáctica en niños que cursaban diferentes niveles de escolaridad, determinándose el porcentaje promedio de aparición de las relaciones interclausulares en la producción oral de cada uno de los grupos etarios. Por otra, describir qué estructuras emergían en mayor o menor grado en cada uno de los niveles de escolaridad estudiados, en dos modalidades discursivas: la narración y la explicación.

La muestra estuvo compuesta por 38 escolares de diferentes estratos socioeconómicos, los cuales se distribuyen en los siguientes niveles de escolaridad: kinder, segundo básico, cuarto básico, sexto básico, octavo básico y cuarto de enseñanza media (último nivel de escolaridad de enseñanza secundaria en Chile). En un primer momento, se les presentó a los participantes dos videos: uno era un cuento creado por el equipo de investigación y el otro trataba de la importancia de la escuela en la sociedad actual. Estos videos duraban dos a tres minutos. Luego, se les exponía al material verbal y gráfico narrativo y, como tarea, se les solicitaba que elaboraran una narración oral basándose en el mismo. Posteriormente, se les exponía al texto oral expositivo con apoyo gráfico y se les solicitaba que elaboraran una explicación oral como si fuera parte de una tarea escolar. Las producciones de los participantes se registraron en una grabadora digital y se transcribieron según las normas de transcripción de Calsamiglia y Tusón (I999), las cuales fueron adaptadas para la ocasión. 
El análisis consistió en la segmentación de paquetes clausulares en la totalidad de lo producido oralmente por los participantes. En base a la clasificación que se presentó en el marco teórico, cada PC fue analizado de acuerdo a las relaciones interclausulares que se encontraban en su interior. El ejemplo de más abajo muestra el tipo de segmentación y análisis que se realizó en la producción de un participante de segundo básico:

Ejemplo I. Segmentación y análisis sintáctico de la secuencia explicativa de un estudiante de $2^{\circ}$ básico

[PCI] En la escuela existen [ISOprin] porque... porque los niños no se podían quedar solos en sus casas [HIPOadvATIPcausal]

[PC2] Como sus padres no se acordaban tanto [HIPOadvATIPcausal] y tenían que ir a trabajar [SIMcoordSIEl] inventaron las escuelas [ISOprin] y por eso son importantes las escuelas [SIMcoordSIEx]

[PC3] La hora más entretenida es el recreo [ISOprin] porque podemos comer y jugar [HIPOadvATIPcausal]

[PC4] Y nos enseñan los profesores [ISOprin] que son inteligentes [ENDOadj] nunca se olvidaron [SIMyuxSIEl].

\section{Análisis de los Resultados}

El análisis de las relaciones sintácticas que produjeron los participantes en cada modalidad discursiva oral se llevó a cabo mediante la descripción de dichas relaciones asignándoles un puntaje que equivale a un índice de complejidad sintáctica. El cálculo estadístico (Pérez, 2010) permitió asignar los siguientes puntajes por tipo de cláusula (Tabla I).

Tabla I. Asignación de puntaje según tipo de relación sintáctica

\begin{tabular}{|c|c|c|}
\hline \multicolumn{2}{|c|}{ TIPO DE RELACIÓN SINTÁCTICA } & PUNTAJE \\
\hline \multicolumn{2}{|l|}{ ISOTAXIS } & I \\
\hline \multirow{2}{*}{ PARATAXIS } & SIMÉTRICA & 4 \\
\hline & ASIMÉTRICA & 8 \\
\hline \multicolumn{2}{|l|}{ HIPOTAXIS } & 16 \\
\hline \multicolumn{2}{|l|}{ ENDOTAXIS } & 24 \\
\hline \multicolumn{2}{|c|}{ NOMINALIZACIÓN } & 24 \\
\hline
\end{tabular}


A partir de los valores asignados a las relaciones interclausulares, se calculó el índice promedio en cada modalidad discursiva producida oralmente por los estudiantes de cada nivel escolar. Los datos arrojados al aplicar este índice en ambas modalidades discursivas se presentan en los siguientes gráficos:

Gráfico I. Índices promedio obtenidos a partir de la producción narrativa.

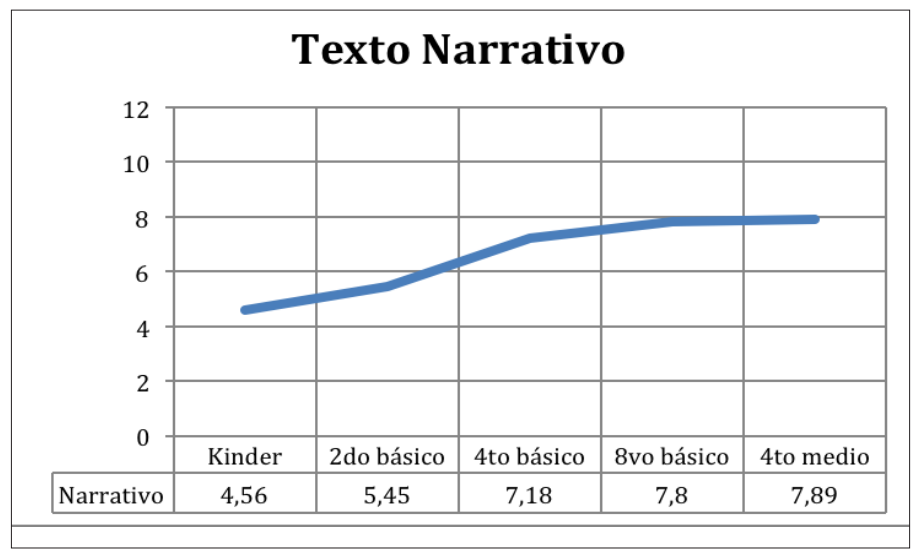

En el Gráfico I, se observa un incremento en el índice de complejidad sintáctica en el discurso narrativo, especialmente entre los primeros años de escolarización. Sin embargo, se observa una meseta en el desempeño sintáctico de los estudiantes de $8^{\circ}$ básico con respecto a los de $4^{\circ}$ de enseñanza media.

Gráfico 2. Índices promedio obtenidos a partir de la producción explicativa.

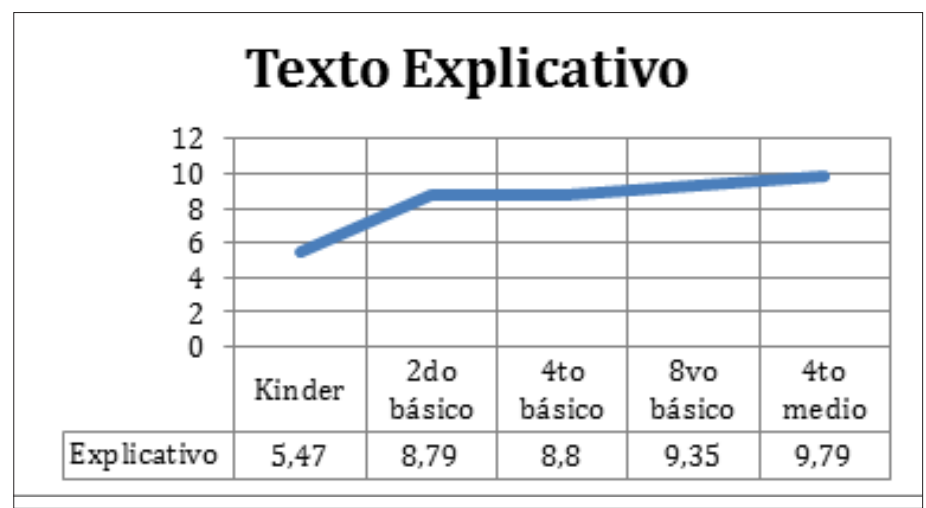


En el Gráfico 2, se observa un incremento en el nivel de complejidad sintáctica en el discurso explicativo, especialmente entre kínder y $2^{\circ}$ básico. Sin embargo, se constata un estancamiento en el desempeño sintáctico de los estudiantes entre $2^{\circ}$ y $4^{\circ}$ básico, un leve incremento entre $4^{\circ}$ y 8 básico, y entre $8^{\circ}$ básico y $4^{\circ}$ medio.

Respecto al uso de las relaciones interclausulares propuestas y medidas en este estudio, el Gráfico 3 da cuenta del porcentaje promedio del uso de dichas categorías sintácticas por nivel educativo.

Gráfico 3. Porcentaje promedio de presencia de la relaciones interclausulares por nivel escolar en la secuencia narrativa

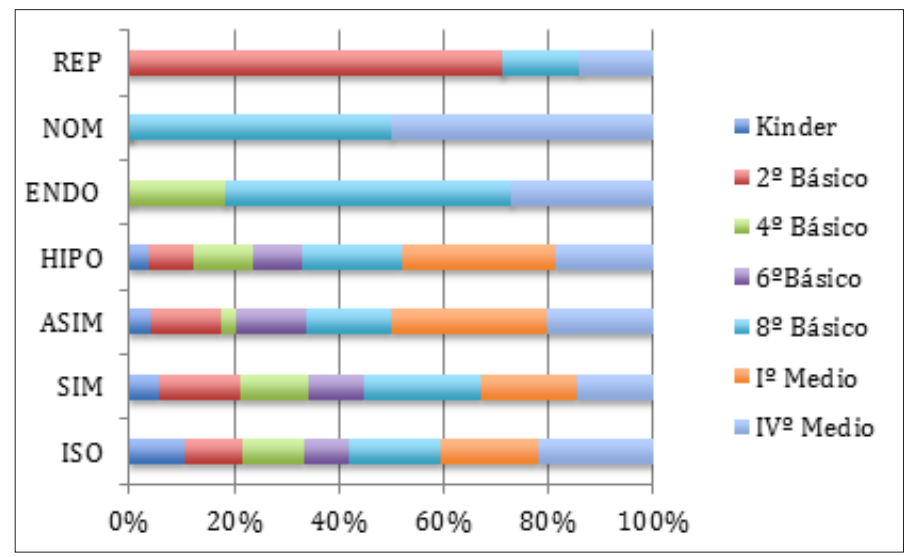

En el Gráfico 3, se observa que, por una parte, la isotaxis, la parataxis y la hipotaxis aparecen en todos los niveles escolares en la producción oral de la secuencia narrativa con mayor o menor grado. Por su parte, la endotaxis se presenta sólo desde cuarto básico en adelante. Asimismo, la nominalización sólo se constató su presencia en dos niveles educativos: octavo básico y cuarto de enseñanza media. Finalmente, la endotaxis reparadora fue una estructura muy utilizada por los estudiantes de segundo básico y, en menor grado, en los escolares de octavo y cuarto de enseñanza media. 
Gráfico 4. Porcentaje promedio de presencia de la relaciones interclausulares por nivel escolar en la secuencia narrativa.

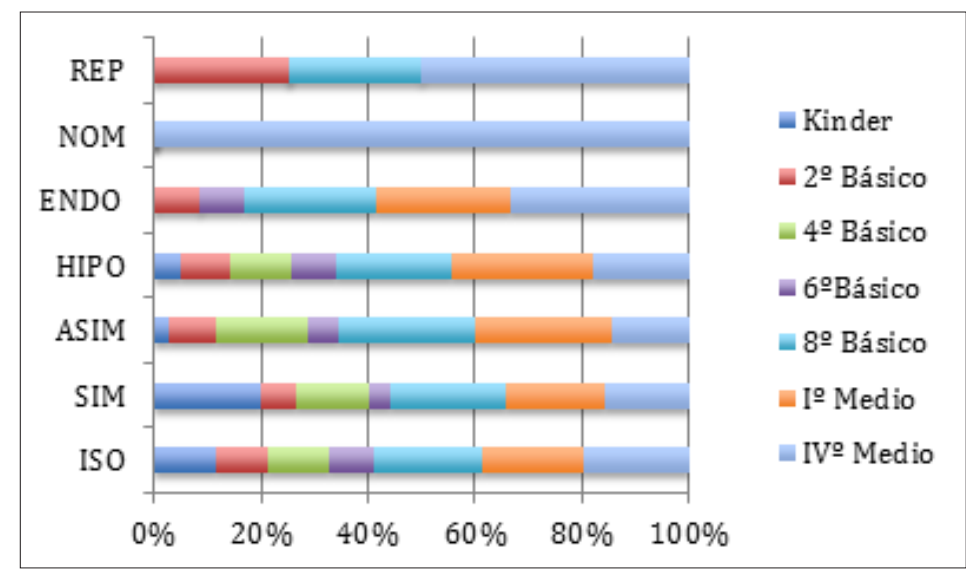

En el Gráfico 4, se observa que, al igual que en la secuencia narrativa, en la secuencia explicativa los participantes de todos los niveles presentan en sus discursos cláusulas isotácticas, paratácticas e hipotácticas. A partir de segundo básico, comienza a aparecer la endotaxis; sin embargo, la nominalización sólo se observa en cuarto de enseñanza media. Llama la atención, que los participantes que cursan cuarto básico no presentan ni endotaxis, ni nominalización, ni endotaxis reparadora, al igual que los participantes de kínder.

Gráfico 5. Presencia absoluta de cada categoría en todos los niveles escolares y en ambas modalidades discursivas

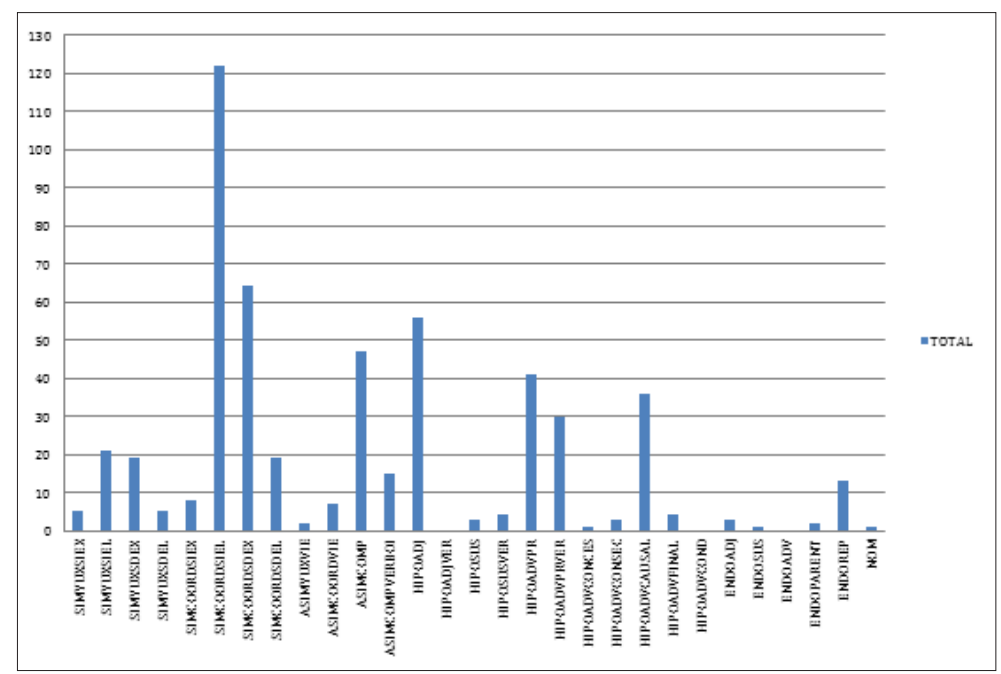


El Gráfico 5 muestra que la categoría interclausular con la mayor presencia en ambas modalidades discursivas y en todos los niveles escolares considerados en este estudio es la cláusula paratáctica simétrica coordinada con sujeto idéntico elidido [SIMcoordSIEL]. Las segundas con mayor presencia son las cláusulas paratácticas simétricas coordinadas con sujeto diferente explícito [SIMcoordSDEx], la hipotaxis adjetiva [HIPOadj] y la paratáctica asimétrica subordinada sustantiva OD [ASIMcomp].

Las relaciones interclausulares que tuvieron menor presencia fueron las paratácticas yuxtapuestas con verbo idéntico elíptico [ASIMyuxvie], las hipotaxis sustantivas [HIPOsus], las hipotaxis adverbiales concesivas, consecutivas y finales; las endotaxis adjetivas y las sustantivas. Finalmente, las que no se presentaron en este estudio fueron las hipoadjetivas verboides, las hipotácticas adverbiales condicionales y las endoadverbiales.

\section{Conclusiones}

Los resultados de este estudio han permitido llegar a varias conclusiones. En primer lugar, existe una relación directamente proporcional entre nivel de escolaridad y complejidad sintáctica, es decir, mientras mayor sea el primero, mayor será la segunda. Dicha relación proporcional se mantiene en la producción de los dos tipos de textos seleccionados para la elicitación oral. La mayor exposición a textos escritos y orales, el incremento en la operatividad cognitiva, el incremento en la familiaridad con sintaxis apropiada al contexto $y$, en fin, la instrucción recibida hacen suponer que las relaciones sintácticas se complejizan a medida que el individuo va madurando.

Asimismo, el índice de complejidad permitió observar que dicho aumento en la narración es más graduado; en cambio, en la secuencia explicativa el incremento es más abrupto en los primeros años escolares y se va estancando en los años posteriores. No se ha encontrado una explicación clara a este fenómeno; sin embargo, otras investigaciones han demostrado que una mayor familiaridad con una estructura textual lleva al sujeto a complejizar su sintaxis (Crespo \& Alvarado, 2013). En base a lo anterior, se postula que la mayor 
frecuencia de la secuencia narrativa en el currículo de lenguaje, permite al alumno dominarla de manera más temprana y a complejizar su sintaxis en forma gradual. La secuencia explicativa, en cambio, aparece con menor frecuencia durante la educación básica; por esta razón, es posible conjeturar que, después de una ganancia considerable en los primeros años (posiblemente debida a la alfabetización inicial), la falta de interacción con esta secuencia textual hace que el estudiante la construya sin complejizar demasiado sus estructuras.

A pesar de esto, si se abandona la perspectiva ontogenética y si se observa los textos producidos por todos los participantes de la muestra en este estudio, es posible afirmar que la arquitectura sintáctica de la secuencia expositiva resulta más compleja que la de la de la secuencia narrativa. Esto se debe, probablemente, a que la secuencia explicativa presenta más dificultades temáticas y discursivas. La actitud del productor de la secuencia explicativa es la de una mayor adaptación de su discurso a las exigencias de esta secuencia, haciendo que éste se mantenga más alejado, más desapegado o más objetivo con respecto a la materia tratada, a diferencia de la actitud que adopta para producir la secuencia narrativa, cuyas exigencias discursivas permiten que el productor se acerque más al tópico en cuestión, sea más subjetivo y le permita representársela con imágenes ficticias. En las secuencias narrativas, por lo general, se encuentran más experiencias personales o más involucramiento personal de parte de los hablantes en la idea y en los eventos de los cuales tienen experiencia o las hayan pensado. Por tanto, el discurso de la secuencia narrativa contiene actitudes más afectivas que las que se podrían encontrar en la explicativa. En otras palabras, a mayor distanciamiento de la materia a tratar pareciera darse mayor complejidad sintáctica en al menos estas dos secuencias textuales.

En segundo lugar, con respecto al uso de relaciones interclausulares, se advirtió que aquellas que denotan un aumento en la complejidad (hipotaxis), se presentan en todos los niveles escolares con diferentes grados de frecuencia. Esto es relevante, pues cláusulas de tipo hipotácticos (supuestamente más tardías) fueron utilizadas por los escolares más pequeños: los participantes de kínder. A partir de allí, puede establecerse que la hipotaxis no deviene después de la 
parataxis, sino que los niños desde pequeños combinan cláusulas coordinadas y subordinadas en sus producciones orales. No obstante este hallazgo, cabe señalar que sí existe una diferencia en el uso de los elementos de los sujetos más pequeños (kínder y segundo básico) respecto de los mayores (cuarto de enseñanza media). En los primeros niveles de enseñanza, existe una tendencia a emplear conectores que permiten yuxtaponer y/o coordinar ideas, y también -aunque con una menor frecuencia- a subordinar o incrustar información. Sin embargo, a medida que aumenta la edad de los participantes, se observa un mayor uso de mecanismos de cohesión como también de elisión del verbo. En otras palabras, la complejidad sintáctica no solo implica ganancia en el aumento de estructuras sintácticas, sino también en la utilización creciente de recursos transoracionales. Los sujetos gramaticales y los pronombres elididos "reflejan" que el referente ha sido presupuesto y que también ayudan a "mantener" el estatus temático, "sirviendo como señal" de que el referente se ha presupuesto al momento de hablar (Levy, 2003). Givón (I979:35) se refiere a este fenómeno como "sintaxización de discurso" y lo define como el cambio desde una "modalidad pragmática" de hablar, caracterizada por una "conjunción suelta" y una proporción de uno a uno de sustantivos y verbos, hacia una "modalidad sintáctica", caracterizada por una "subordinación apretada" y una proporción sustantivo a verbo más amplia. El autor también señala que existe la posibilidad de homologar los cambios ontogenéticos en el discurso, los cuales ocurren diacrónicamente, en el sentido de que los procesos que parecen ocurrir a medida que el niño modifica su modalidad comunicativa a través de la sintaxización son idénticos a los procesos que crean estructuras sintácticas en el lenguaje a través del tiempo.

Ahora bien, respecto a la nominalización, por la cual un verbo es reemplazado por un sustantivo, sólo fue producida por los participantes de $8^{\circ}$ básico y de cuarto de enseñanza media en la secuencia explicativa y sólo por los de cuarto de enseñanza media en la narración, pero con una frecuencia de uso muy baja respecto a las estructuras construidas con recursos sintácticos. Estos resultados permiten suponer que -tal como lo comprobó Berman (2004)-, los jóvenes adultos emplean nominalizaciones para formular generalizaciones, distanciarse del tópico y expresar su posición 
frente a éste, en maneras que no son posibles de encontrar entre los participantes más jóvenes. Es posible adjudicar estos cambios, no solo al mejor dominio lingüístico del sujeto sino también a que la nominalización es una estructura frecuente en el lenguaje de las clases y de los textos de estudio (Marín, 2005) y, por lo tanto, es explicable una mayor familiarización con ellos a medida que el individuo empieza a usar la lectura como una herramienta de aprendizaje.

Finalmente, se puede concluir que, si bien lo observado por el presente estudio es preliminar, ha permitido señalar el camino a seguir para describir la complejidad sintáctica creada por niños y jóvenes chilenos. Los procedimientos han sido efectivos, la metodología de trabajo ha sido comprobada y la asignación de puntaje ha sido validada en forma estadística. Se espera que el estudio mayor no sólo sirva para observar la complejidad sintáctica en la muestra seleccionada, sino que también arroje resultados lo suficientemente confiables y generales como para proporcionar implicaciones pedagógicas. Todavía queda por observar si las mismas prácticas orales para complejizar la sintaxis en la producción oral ocurren también en la expresión escrita en los mismos participantes o, al menos, en participantes del mismo nivel de escolaridad.

\section{Referencias bibliográficas}

Adam, J. M. (I992): "Les textes: types et prototypes. Récit, description, argumentation, explication et dialogue”, París, Nathan.

Adam, J, M. (1996): "(Proto)tipos: La estructura de la composición en los textos”, Textos de Didáctica de la Lengua y la Literatura, I0, 9-22.

Bartolomé, R. (2008): "La adquisición y el aprendizaje de los modificadores nominales en el discurso escrito de estudiantes de español como LI y L2”, Revista Electrónica de Lingüística Aplicada, 7, págs. 45-54.

Berman, R. \& Slobin, D. (1994): "Relating events in narrative: A crosslinguistic developmental study”, New Jersey, Lawrence Erlbaum Associates.

Berman, R. (1996): "Form and function in developing narrative skills", en D. Slobin, J. Gerhardt, A. Kyratzis, y J. Guo (eds.), Social interaction, social context, and language, New Jersey, Lawrence Erlbaum Associates, págs. 343-367. 
Berman, R. (2004): "Introduction: Developing discourse stance in different text types and languages", Journal of Pragmatics, 37, 105-124.

Brunner, J. (I990): Acts of meaning, Cambrigde, MA, Harvard University Press.

Cahana-Amitay, D. \& Sandback, A. (2000): "Clause packaging in written expository texts”, en M. Aparici, N. Argerich, J. Perera, E. Rosado y L. Tolchinsky (eds.), Developing literacy across genres, modalities, and languages, Barcelona, Universidad de Barcelona, págs. 129- 148.

Chafe, W. (1994): Discourse, consciousness, and time: The flow and displacement of conscious experience in speaking and writing, Chicago, Chicago University Press.

Coloma, C.; Peñaloza, C. \& Fernández, R. (2007): "Producción de oraciones complejas en niños de 8 y I0 años”, Revista de Lingüística Teórica y Aplicada, 45, págs. 33-44.

Crespo, N. y Alvarado, C. "Desarrollo sintáctico a través de la escolaridad: una medición a partir de la diversidad clausular”, Revista Logos, en prensa.

Gee, J. (I989): "Two styles of narrative construction and their linguistic and educational implications”, Boston University Journal of Education, 17, págs. $79-175$.

Givón, T. (1979): On understanding grammar, Nueva York, Academic Press.

Givón, T. (I985): "Iconicity, isomorphism, and non-arbitrary coding in syntax”. En J. Haiman (ed.), Iconicity in syntax, Amsterdam: Jobn Benjamins, págs. $187-219$.

Halliday, M. (1993): An introduction to functional grammar, London, Edward Arnold.

Heurly, L. (1997): "Processing units in written texts: Paragraphs or information blocks?”, en J. Costerman \& M. Fayol (eds), Processing interclausular relationships, studies in production and comprehension of text, Amsterdam, Lawrence Erlbaum Associates, págs. 179-200.

Hunt, K. (1970): "Recent measures in syntactic development", en M. Lester (ed.), Readings in applied transformational grammar, Nueva York, Holt, Rinebart and Winston, págs. 187-200.

Katzenberger, I. (I999): "Further ideas on 1(onger)-u(nits)", en R. Aiseman (ed): Working papers in developing literacy across genres, modalities, and languages, Tel-Aviv, Tel-Aviv University, págs. 128 14l.

Katzenberger, I. (2004): "The development of clause packaging in spoken and written texts", Journal of Pragmatics, 36, págs. 192 l-1948.

Levy, E. (I999): A social-pragmatic account of the development of planned discourse. Human Development, 42, 225-246. 
Levy, E. (2003): "The roots of coherence in discourse", Human Development, 46, págs. 169- 188.

Nir-Sagiv, B. \& Berman, R. (2010): "Complex syntax as a window on contrastive rhetoric", Journal of Pragmatics, 42, págs. 744-765.

Ochs, E. (1979): "Planned and unplanned discourse", En T. Givón (eds.), Syntax and semantics, Nueva York, Academic Press, págs. 5I-80.

Pérez, L. (2010): "Informe cálculos de puntajes de relaciones interclausulares", documento interno no publicado, Proyecto FONDECYT No I I00600, Universidad Católica de Valparaíso, Chile.

Scinto, L. (1984): "The architectonics of texts produced by children and the development of higher cognitive functions”, Discourse Processes, 7, págs. $371-418$.

Tolchinsky, L. \& Rosado, E. (2005): "The effect of literacy, text type, and modality on the use of grammatical means for agency alternation in Spanish”, Journal of Pragmatics, 37, págs. 209-237.

Thompson, G. (2004): Introducing functional grammar, London, Arnold.

Véliz, M. (1988): "Evaluación de la madurez sintáctica en el discurso”, Revista de Lingüística Teórica y Aplicada, 26, págs. I05-I4I.

Véliz, M. (I999): “Complejidad sintáctica y modos del discurso”, Estudios Filológicos, 34, págs. I8I-I92. 